\title{
Helicity Entanglement of Moving Bodies
}

\author{
Shuxin Shao \\ Department of Physics, Beijing Normal University, Beijing, 100875, China \\ Song $\mathrm{He}$ \\ School of Physics, Peking University, Beijing, 100871, China
}

\author{
Hongbao Zhang* \\ Perimeter Institute for Theoretical Physics, Waterloo, Ontario, N2L 2Y5, Canada \\ Department of Applied Mathematics, University of Waterloo, Waterloo, Ontario, N2L 3G1, Canada
}

(Dated: October 27, 2018)

\begin{abstract}
We investigate the Lorentz transformation of the reduced helicity density matrix for a pair of massive spin $\frac{1}{2}$ particles. The corresponding Wootters concurrence shows no invariant meaning, which implies that we can generate helicity entanglement simply by the transformation from one reference frame to another. The difference between the helicity and spin case is also discussed.
\end{abstract}

As a central concept, quantum entanglement is the major resource in quantum information science such as quantum teleportation and quantum computation [1]. Therefore in quantum information science, one of the most critical issues is how to generate quantum entanglement. In general, quantum entangled states are prepared through some kinds of dynamical processes. However, it is recently shown in the domain of relativistic quantum information that quantum entanglement has somewhat observer dependent property [2]. Speaking specifically, for a single free massive spin $\frac{1}{2}$ particle, the spin entropy, measurement of entanglement between spin and momentum degrees of freedom, has no invariant meaning under the transformation of inertial reference frames $[3]$. In particular, even though the initial state is a direct product of a function of momentum and a function of spin, the Lorentz transformed state is not a direct product, which means that spin and momentum appear to be entangled. Nonetheless, this is not the familiar type of entanglement available for quantum communication, because both degrees of freedom belong to the same particle, rather than to the distinct subsystems that could be widely separated. Based on this observation, Gingrich and Adami have investigated the Lorentz transformation properties of entanglement between a pair of massive spin $\frac{1}{2}$ particles [4]. As a result, while the entanglement of the two particles' entire wave function, i.e., both momentum and spin included, is Lorentz invariant, their spin or momentum entanglement may change due to Lorentz transformation. Thus in some sense only by transforming one reference frame to another does relativity provide a brand new alternative road to create quantum entanglement, which could be used for entanglement manipulation.

As inferred above, in relativistic quantum information, previous investigations focus primarily on the spin relevant entanglement [5, 6, 7, 8, 9, 10, 11]. But it is the helicity rather than spin that is more often under both theoretical consideration and experimental detection in high energy physics, because the helicity has an advan- tage in providing a smooth transition to the massless case. Although both the helicity states and spin states can constitute the basis of Hilbert space of one particle, they differ in the way of unitary transformation under the action of Lorentz group [12]. As analyzed most recently for a single free massive spin $\frac{1}{2}$ particle, the entanglement properties for helicity differ remarkably from those for spin after we trace out the momentum degree of freedom under Lorentz transformation [13]. Nevertheless, obviously it is more intriguing and significant to investigate the Lorentz transformation properties for helicity entanglement between a pair of massive spin $\frac{1}{2}$ particles, which is just what we shall report in this paper.

Given a field with mass $m$ and spin $\frac{1}{2}$, we can also construct the helicity states $|p ; \lambda\rangle$ as a complete orthonormal basis for Hilbert space of one particle. Associated with a Lorentz transformation $\Lambda$ the unitary operator $U(\Lambda)$ acting on these helicity states gives 12]

$$
\begin{aligned}
& U(\Lambda)|p ; \lambda\rangle \\
& =\sqrt{\frac{(\Lambda p)^{0}}{p^{0}}} D_{\lambda^{\prime} \lambda}\left[R^{-1}(\Lambda p) L^{-1}(\Lambda p) \Lambda L(p) R(p)\right]\left|\Lambda p ; \lambda^{\prime}\right\rangle \\
& =\sqrt{\frac{(\Lambda p)^{0}}{p^{0}}} D_{\lambda^{\prime} \lambda}\left[B^{-1}(\Lambda p) R^{-1}(\Lambda p) \Lambda R(p) B(p)\right]\left|\Lambda p ; \lambda^{\prime}\right\rangle \\
& =\sqrt{\frac{(\Lambda p)^{0}}{p^{0}}} D_{\lambda^{\prime} \lambda}[Z(\Lambda, p)]\left|\Lambda p ; \lambda^{\prime}\right\rangle .
\end{aligned}
$$

Here $R(p)$ is the rotation that carries the $z$ axis into the direction $\mathbf{p}, B(p)$ is the boost from rest to the momentum $|\mathbf{p}|$ in the $z$ direction, and $L(p)$ is the pure boost from rest to the momentum $\mathbf{p}$. Obviously, $L^{-1}(\Lambda p) \Lambda L(p)$ is just Wigner rotation, usually denoted by $W(\Lambda, p)$. In addition, $D$ is the spin $\frac{1}{2}$ irreducible unitary representation of Lorentz group. Note that these helicity states differ in way of unitary transformation from spin states under the action of Lorentz group, since under Lorentz transformations spin states change accord- 
ing to Wigner rotation, which is related to $Z(\Lambda, p)$ as $Z(\Lambda, p)=R^{-1}(\Lambda p) W(\Lambda, p) R(p)$.

Thus a pure state for two massive spin $\frac{1}{2}$ particles can be expanded in terms of the helicity states as

$$
|\Psi\rangle=\int d \mathbf{p} d \mathbf{q} \sum_{\lambda \sigma} g_{\lambda \sigma}(\mathbf{p}, \mathbf{q})|p ; \lambda\rangle|q ; \sigma\rangle,
$$

with the normalized condition

$$
\int d \mathbf{p} d \mathbf{q} \sum_{\lambda \sigma}\left|g_{\lambda \sigma}(\mathbf{p}, \mathbf{q})\right|^{2}=1
$$

Then the corresponding reduced helicity density matrix can be obtained by tracing out the momentum degrees of freedom, i.e.,

$$
\begin{aligned}
\rho & =\operatorname{Tr}_{\mathbf{p}, \mathbf{q}}[|\Psi\rangle\langle\Psi|]=\int d \mathbf{p} d \mathbf{q}\langle\mathbf{p}, \mathbf{q} \mid \Psi\rangle\langle\Psi \mid \mathbf{p}, \mathbf{q}\rangle \\
& =\int d \mathbf{p} d \mathbf{q} \sum_{\lambda \tilde{\lambda} \sigma \tilde{\sigma}}\left[g_{\lambda \sigma}(\mathbf{p}, \mathbf{q}) g_{\tilde{\lambda} \tilde{\sigma}}^{*}(\mathbf{p}, \mathbf{q})|\lambda, \sigma\rangle\langle\tilde{\lambda}, \tilde{\sigma}|\right]
\end{aligned}
$$

where we have employed the orthonormal relation for the helicity states.

The entanglement between the helicity degrees of freedom can be quantified by calculating Wootters concurrence 14]

$$
C(\rho)=\operatorname{Max}\left\{k_{1}-k_{2}-k_{3}-k_{4}, 0\right\},
$$

where $\left\{k_{1} \geq k_{2} \geq k_{3} \geq k_{4}\right\}$ are the square roots of the eigenvalues of the matrix $\rho \tilde{\rho}$ with the time reversed matrix

$\tilde{\rho}=\left[\left(\begin{array}{cc}0 & -i \\ i & 0\end{array}\right) \otimes\left(\begin{array}{cc}0 & -i \\ i & 0\end{array}\right)\right] \rho^{*}\left[\left(\begin{array}{cc}0 & -i \\ i & 0\end{array}\right) \otimes\left(\begin{array}{cc}0 & -i \\ i & 0\end{array}\right)\right]$.

Note that under a Lorentz transformation $\Lambda$ the two particle state changes as follows

$$
\begin{aligned}
& \left|\Psi^{\prime}\right\rangle=U(\Lambda)|\Psi\rangle=\int d \mathbf{p} d \mathbf{q} \sum_{\lambda \lambda^{\prime} \sigma \sigma^{\prime}} \sqrt{\frac{(\Lambda p)^{0}}{p^{0}}} \sqrt{\frac{(\Lambda q)^{0}}{q^{0}}} \\
& g_{\lambda \sigma}(\mathbf{p}, \mathbf{q}) D_{\lambda^{\prime} \lambda}[Z(\Lambda, p)] D_{\sigma^{\prime} \sigma}[Z(\Lambda, q)]\left|\Lambda p ; \lambda^{\prime}\right\rangle\left|\Lambda q ; \sigma^{\prime}\right\rangle .
\end{aligned}
$$

Accordingly one can obtain the transformed reduced helicity density matrix as

$$
\begin{aligned}
& \rho^{\prime}=\int d \mathbf{p} d \mathbf{q} \sum_{\lambda \lambda^{\prime} \sigma \sigma^{\prime}}^{\tilde{\lambda} \tilde{\lambda}^{\prime} \tilde{\sigma} \tilde{\sigma}^{\prime}} D_{\lambda^{\prime} \lambda}[Z(\Lambda, p)] D_{\sigma^{\prime} \sigma}[Z(\Lambda, q)] g_{\lambda \sigma}(\mathbf{p}, \mathbf{q}) \\
& g_{\tilde{\lambda} \tilde{\sigma}}^{*}(\mathbf{p}, \mathbf{q}) D_{\tilde{\lambda} \tilde{\lambda}^{\prime}}^{\dagger}[Z(\Lambda, p)] D_{\tilde{\sigma} \tilde{\sigma}^{\prime}}^{\dagger}[Z(\Lambda, q)]\left|\lambda^{\prime}, \sigma^{\prime}\right\rangle\left\langle\tilde{\lambda}^{\prime}, \tilde{\sigma}^{\prime}\right|
\end{aligned}
$$

By Eq.(1), $D[Z(\Lambda, p)]$ is always an identity matrix if $\Lambda$ is a purely spacial rotation transformation, which means that the reduced helicity matrix is completely the same among those inertial observers without relative motion but with different identification of spacial direction. This property stems essentially from the fact that the helicity $\frac{(\mathbf{p} \cdot \mathbf{S})}{|\mathbf{p}|}$ remains invariant under a purely spacial rotation transformation, where $S$ denotes the spin [15]. Note that any Lorentz transformation can always be decomposed into the product of a pure boost and a pure rotation. We thus shall concentrate on what happens to the reduced helicity matrix and the corresponding Wootters concurrence when $\Lambda$ is a pure boost transformation. Furthermore, the pure boost transformations are similarly equivalent with one another by rotations, which means the reduced helicity density matrix only depends on the speed of relative motion between the inertial observers. Therefore we shall only need to consider the pure boost transformations along the $z$ axis.

In the special case mentioned above, set

$$
\Lambda=\left(\begin{array}{cccc}
\cosh \eta & 0 & 0 & \sinh \eta \\
0 & 1 & 0 & 0 \\
0 & 0 & 1 & 0 \\
\sinh \eta & 0 & 0 & \cosh \eta
\end{array}\right), \eta \leq 0
$$

and

$$
p=m[\cosh \tau, \sinh \tau(\sin \theta \cos \phi, \sin \theta \sin \phi, \cos \theta)], \tau \geq 0,
$$

then employing Eq.(1), we obtain

$$
\begin{aligned}
& D[Z(\Lambda, p)]=\left(\begin{array}{cc}
e^{-\frac{\alpha}{2}} & 0 \\
0 & e^{\frac{\alpha}{2}}
\end{array}\right) \times \\
& \left(\begin{array}{cc}
\cos \frac{\beta}{2} & \sin \frac{\beta}{2} \\
-\sin \frac{\beta}{2} & \cos \frac{\beta}{2}
\end{array}\right)\left(\begin{array}{cc}
e^{\frac{\eta}{2}} & 0 \\
0 & e^{-\frac{\eta}{2}}
\end{array}\right) \times \\
& \left(\begin{array}{cc}
\cos \frac{\theta}{2} & -\sin \frac{\theta}{2} \\
\sin \frac{\theta}{2} & \cos \frac{\theta}{2}
\end{array}\right)\left(\begin{array}{cc}
e^{\frac{\tau}{2}} & 0 \\
0 & e^{-\frac{\tau}{2}}
\end{array}\right),
\end{aligned}
$$

where $\alpha$ and $\beta$ satisfy

$$
\cosh \alpha=\cosh \eta \cosh \tau+\sinh \eta \sinh \tau \cos \theta, \alpha \geq 0,
$$

and

$\cos \beta=\frac{\sinh \eta \cosh \tau+\cosh \eta \sinh \tau \cos \theta}{\sqrt{\sinh ^{2} \tau \sin ^{2} \theta+(\sinh \eta \cosh \tau+\cosh \eta \sinh \tau \cos \theta)^{2}}}$

with $\pi \geq \beta \geq 0$, respectively.

For simplicity but without loss of generalization, choose in particular the initial normalized state with the nonvanishing component as follows

$$
g_{\frac{1}{2} \frac{1}{2}}(\mathbf{p}, \mathbf{q})=f(\mathbf{p}) f(\mathbf{q}),
$$

where the momentum distribution function $f$ is taken as a Gaussian. i.e.,

$$
f(\mathbf{p})=\pi^{-\frac{3}{4}} \varepsilon^{-\frac{3}{2}} e^{-\frac{\mathbf{p}^{2}}{2 \varepsilon^{2}}}
$$




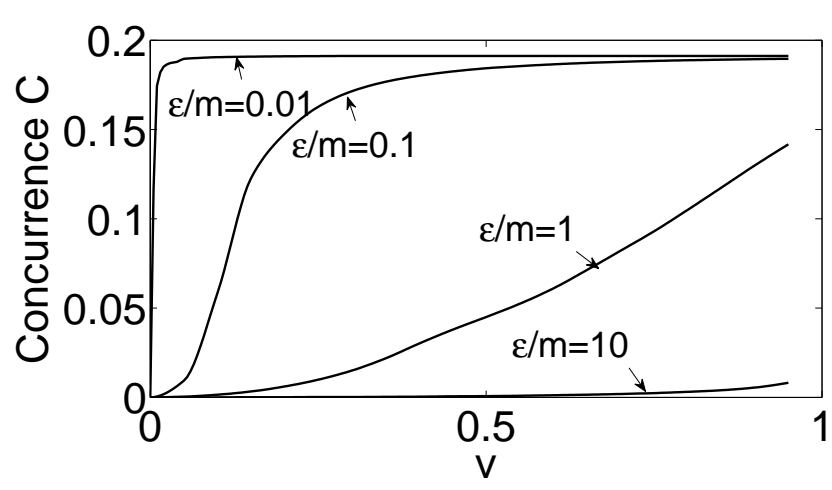

FIG. 1: The Wootters concurrence $C$ as a function of the speed $v=-\tanh \eta$ of the inertial observers with respect to the original inertial reference frame.

with the distribution width $\varepsilon$. It is obvious that this initial state is an unentangled helicity state, and its Wootters concurrence vanishes. However, with respect to the moving inertial observers, the corresponding concurrence is greater than zero in general, which is carried out by numerical calculations and is specifically illustrated in Fig[1.

As shown in Fig 1, with the speed of the inertial observers increasing, the variation behavior of the corresponding concurrence is dependent upon the width-mass ratio $\frac{\varepsilon}{m}$. In particular, for the large width-mass ratio limiting case, the resultant concurrence varies pretty slightly and remains nearly invariant. On the other hand, for the limiting case of sharp momenta which corresponds to the small width-mass ratio, the concurrence blows up from zero and rapidly saturates. Speaking specifically, it arrives at a constant value at small velocities of the observers, and then remains nearly invariant regardless of the increase of speed of the observers. So, if we restrict ourselves to helicity measurements in this case, an observer in the original inertial reference frame cannot use quantum entanglement as a resource while the moving observers can, simply with small velocities. This is remarkably different from the spin case, since the concurrence for spin always remains invariant in the limit of sharp momenta [4, 5, 6] .

The crucial physics underlying this behavior lies in the following fact: With the smaller width-mass ratio $\frac{\varepsilon}{m}$, i.e., sharper momentum distribution, the helicity of the prepared particles becomes more sensitive to Lorentz boost, due to the concentration of its momentum in a smaller neighborhood around zero. To put it another way, for the case of smaller width-mass ratio, the smaller speed of observers is needed to make the flip of helicity from right to left saturate such that the corresponding concurrence approaches the saturated value.
In summary, the transformation of the reduced helicity density matrix under Lorentz group is investigated for a pair of massive spin $\frac{1}{2}$ particles. Especially, we have calculated the corresponding Wootters concurrence for an initial unentangled helicity state by numerical computation. Our results show that the corresponding concurrence is not a Lorentz invariant scalar, which thus opens a new alternative way to generate quantum entanglement between helicity degrees of freedom. In addition, as the speed of the inertial observers increases, the specific variation of concurrence for the helicity demonstrates a distinct behavior from the spin case, which essentially originates from the fact that the helicity states differ significantly from the spin states in the transformation property under Lorentz boost, as pointed out in the beginning.

HZ is grateful to helpful suggestions from Achim Kempf. He would also like to give much thanks to Robin Blume-Kohout for valuable communication. Work by SH was supported by NSFC(nos.10235040 and 10421003). $\mathrm{HZ}$ was supported in part by the Government of China through CSC(no.2007102530). This research was supported by Perimeter Institute for Theoretical Physics. Research at Perimeter Institute is supported by the Government of Canada through IC and by the Province of Ontario through MRI.

* Electronic address: hzhang@perimeterinstitute.ca

[1] The Physics of Quantum Information, edited by D. Bouwmeester et al.(Springer-Verlag, 2000).

[2] A. Peres and D. R. Terno, Rev. Mod. Phys. 76: 93(2004).

[3] A. Peres et al., Phys. Rev. Lett. 88: 230402(2002).

[4] R. M. Gingrich and C. Adami, Phys. Rev. Lett. 89: 270402(2002).

[5] P. M. Alsing and G. J. Milburn, quant-ph/0203051

[6] J. Pachos and E. Solano, Quant. Inf. Comput. 3: 115(2003).

[7] P. Caban and J. Rembielinski, Phys. Rev. A 72: 012103(2005).

[8] L. Lamata et al., Phys. Rev. Lett. 97: 250502(2006).

[9] T. F. Jordan et al., Phys. Rev. A 73: 032104(2006).

[10] L. Lamata et al., Phys. Rev. A 73: 052325(2006).

[11] T. F. Jordan et al., quant-ph/0608061.

[12] S. Weinberg, in Lectures on Particles and Field Theory, vol. II of Lectures delivered at Brandeis Summer Institute in Theoretical Physics, edited by S. Deser and F. W. Ford(Prentice-Hall, Inc., Englewood Cliffs, 1964).

[13] S. He et al., J. Phys. A 40: F857(2007).

[14] W. K. Wootters, Phys. Rev. Lett. 80: 2245(1998).

[15] Note that the reduced spin density matrix changes as $\rho^{\prime}=[D(\Lambda) \otimes D(\Lambda)] \rho\left[D^{\dagger}(\Lambda) \otimes D^{\dagger}(\Lambda)\right]$ for a purely spacial rotation transformation $\Lambda$. However, the corresponding concurrence remains invariant under such a rotation. 